\title{
Tune Tracking with a PLL in the Tevatron
}

\author{
C.Y. $\operatorname{Tan}^{1}$ \\ Fermi National Accelerator Laboratory, Accelerator Division/Tevatron, MS 341, \\ P.O. Box 500, Batavia, IL 60510, USA
}

\begin{abstract}
The Tevatron tune tracker is based on the idea that the phase of the transverse frequency response of the beam can be measured quickly and accurately enough so that the phase at the betatron tune resonance can be tracked by a phase locked loop (PLL). In this paper, a mathematical model of this idea is discussed and is used as the basis for the realization of the tune tracker hardware. The tune tracker has been successfully tested under different beam conditions and is now operational in the Tevatron.
\end{abstract}

Key words:

Tevatron, tune tracker, tune tracking, PLL, phase locked loop

\section{Introduction}

The Tevatron which operates at $980 \mathrm{GeV}$ is the highest energy colliding machine in world. Since 2001, the ongoing Run II programme has collected Email address: cytan@fnal.gov (C.Y. Tan).

1 funded by DOE under contract DE-AC02-76CH03000 and the US-LARP collaboration. 
more than $2 \mathrm{fb}^{-1}$ of integrated luminosity ${ }^{2}$ for two experiments from the collisions of $36 \times 36$ bunches of protons and anti-protons. In normal high energy physics (HEP) conditions, each proton bunch contains $(200-300) \times 10^{9}$ particles while each anti-proton bunch contains $(20-30) \times 10^{9}$ particles. The two beams of protons and anti-protons counter-rotate in the same 4 miles of beam pipe for the duration of a store which can last between 20 to 30 hours. In order to prevent the protons and anti-protons from colliding away from the interaction points at the experiments, electrostatic separators are used to separate them on mirrored orbits called a helix. The injection energy of the Tevatron is at $150 \mathrm{GeV}$ and after the protons and anti-protons are injected, they are accelerated (called ramped) to $980 \mathrm{GeV}$ in 85 seconds. The transverse spot size of the beam at the experiments is then reduced from $66 \mu \mathrm{m}$ to $30 \mu \mathrm{m}$ in a process called the squeeze. The smaller spot size increases the transverse particle density and thus increases the number of collisions at the experiments. Two important parameters in this entire process are the horizontal and vertical betatron tunes (or simply called tunes). The tunes are chosen so that they are far from resonances which can cause poor beam lifetimes and high losses. For the Tevatron at $150 \mathrm{GeV}$, the revolution frequency is $f_{r}=47.712 \mathrm{kHz}$ and the nominal horizontal and vertical tunes are $\nu_{x}=0.583\left(\nu_{x} f_{r}=27.816 \mathrm{kHz}\right)$ and $\nu_{y}=0.575\left(\nu_{y} f_{r}=27.434 \mathrm{kHz}\right)$ respectively. Control of these tunes to \pm 0.001 or better is necessary for the survival of the beam during the ramp and squeeze process.

The goal of the Tevatron tune tracker is a Run II project is to be able to track the tunes up the ramp and through the squeeze for every store. The ultimate goal is to eventually build a tune feedback system based on

\footnotetext{
$\overline{2}$ as of 14 Nov 2005 for the two experiments CDF and D0.
} 
the tune tracker in order to control them up the ramp. The idea behind the tune tracker is that the transverse frequency response of the beam can be quickly measured and that the phase null of the response is at the peak of the resonance. See Figure 1. By following this null, any motion of the resonance peak can be tracked. Tracking with this method is called the PLL (phase locked loop) method. An added complication in tracking the tune is that for bunched beams, the bunch also executes phase motion w.r.t. accelerating RF (radio frequency). This shows up as sidebands around the tune and are called the synchrotron sidebands. These synchrotron sidebands can also have phase nulls which can make the PLL lock to a phase null which is not at the betatron tune. The distance between the synchrotron sidebands is called the synchrotron tune. For the Tevatron, the synchrotron tune is $85 \mathrm{~Hz}$ at $150 \mathrm{GeV}$ and $34 \mathrm{~Hz}$ at $980 \mathrm{GeV}$.

Tune tracking with a PLL has also been implemented at RHIC (Relativistic High Energy Ion Collider) at Brookhaven National Laboratory (BNL)[1]. The PLL designed for the Tevatron is different from the BNL PLL in that it has the capability of exciting the beam at three different synchrotron frequencies around the tune or exciting the beam with a pulsed sine wave. The first method is called multi-tone continuous excitation PLL (or continuous PLL for short) and the second method is called pulsed excitation PLL (or pulsed PLL for short). In this paper, only the results from single tone continuous and pulsed PLLs will be presented. 


\section{Theory}

The response of the PLL tune tracker can be solved analytically if the beam frequency response is first approximated by a damped simple harmonic oscillator and then its phase response linearised. It is well known that for a simple harmonic oscillator at resonance $\omega_{0}=\dot{\Theta}_{0}$, its phase is shifted by $-90^{\circ}$ w.r.t. the excitation. It is easier to visualize the locking process if the entire phase response is shifted by $+90^{\circ}$ so that the zero crossing of the phase is at the peak of the magnitude response. See Figures 2(a) and (b). The linearisation of the phase response can take place if the excitation is never too far away from the zero crossing which means that it can be approximated by a straight line with slope $-K$ s. See Figure 2(c). With this approximation, the output phase $\Theta_{\text {out }}(t)$ is related to the excitation phase $\Theta_{\text {in }}(t)$ by a linear phase shift

$$
\Theta_{\text {out }}=\Theta_{\text {in }}-K\left(\dot{\Theta}_{\text {in }}-\dot{\Theta}_{0}\right)
$$

Using the approximation of the phase response from equation (1) and the block diagram of the PLL shown in Figure 3, the closed loop response $\mathcal{H}$ is

$$
\mathcal{H}(s) \equiv \frac{\theta_{2}}{\theta_{0}}=\frac{g K_{0} K_{d} K G(s)}{s+g K_{0} K_{d} K G(s)}
$$

where $\theta_{2,0}(s)$ is the Laplace transform of $\Theta_{2,0}(t), g$ is the gain multiplier in units of $\mathrm{s}^{-1}, K_{0}$ is the gain from the voltage controlled oscillator (VCO) in units of rad. $\mathrm{s}^{-1} / \mathrm{V}, K_{d}$ is the gain in the phase detector in units of $\mathrm{V} / \mathrm{rad}$, and $G(s)$ is a low pass filter response function and is dimensionless. These units ensure that $\mathcal{H}$ is dimensionless as required. 
The natural definition of the error $\theta_{e}$ in the phase lock is given by

$$
\theta_{e}=\theta_{0}-\theta_{2}
$$

because this relates the phase error between the beam response phase null to the phase of the excitation. By using equations (1) and (2), this leads to the error transfer function given by $\mathcal{H}_{e}$

$$
\mathcal{H}_{e} \equiv \frac{\theta_{e}}{\theta_{0}}=\frac{s}{s+g K_{0} K_{d} K G(s)}
$$

With this result, it can be shown that when $G(s)$ is a passive lag filter and there is a step in the beam response phase or beam response resonant frequency the PLL will eventually lock to the phase null with no error. For the case when the beam response resonant frequency is ramped, the PLL will lock to the ramp with an error inversely proportional to the gain of the loop $g K_{0} K_{d} K[2]$.

\subsection{Pulsed Excitation}

The motivation behind using pulsed excitation rather than a continuous sine wave excitation comes from the observation that there are phase nulls associated with each synchrotron line that are superimposed over a betatron phase response. See Figure 1(a). In principle, the continuous PLL can lock to any of these synchrotron lines. See Figure 1(b). In order to overcome this problem and to always lock to the background betatron phase null, the effect of these extra synchrotron phase nulls must some how be removed. One solution is to pulse the beam rather than continuously exciting the beam. Pulsing the

excitation will spread out the zero bandwidth sine wave to a finite bandwidth 
$\Delta_{\text {bw }}$ given by

$$
\Delta_{\text {bw }}(\mathrm{Hz})=\frac{2}{\tau}
$$

where $\tau$ is the width of the pulse.

For example, in the Tevatron at $150 \mathrm{GeV}$, the synchrotron frequency is about $85 \mathrm{~Hz}$. If $\tau=10 \mathrm{~ms}$ then $\Delta_{\mathrm{bw}}=200 \mathrm{~Hz}$ from equation (5). This means that the phase response from exciting the beam with pulses will be averaged over two synchrotron lines. The "off" time between pulses that is sufficient to smooth out the synchrotron lines to reveal the background betatron tune is found empirically to be $30 \mathrm{~ms}$ and is shown in Figure 5.

\section{Implementation}

The PLL tune tracker system is shown in in Figure 6. The system can be divided into four major parts

(1) Software PLLs. A digital signal processor (DSP) implements the software PLLs which consists of the phase detector, integrator and filter shown in Figure 3. The DSP is a Texas Instruments TMS320C6416 fixed point processor running at $500 \mathrm{MHz}$. This DSP is just fast enough to execute the software code of three PLLs when each PLL is sampling at $100 \mathrm{kHz}$.

(2) Betatron Tune Detector. The betatron tune detector is a resonant $21.4 \mathrm{MHz}$ Schottky pickup. The Schottky pickup is extremely sensitive to coherent and incoherent motion of the beam and is the workhorse for measuring tunes in the Tevatron. This pickup has been designed to be directional so that anti-proton tunes will not affect the measurement of the proton 
tunes. The detector's resonant frequency was selected to be $21.4 \mathrm{MHz}$ because commercially available crystal filters which are narrow band can be used to filter out the revolution harmonics[3] which otherwise would swamp any downstream amplifier. Downstream of the detector is an automatic gain control (AGC) circuit which helps preserve the dynamic range of the analogue to digital converter (ADC) by keeping fixed the size of the signal going into it.

(3) Excitation. The excitation comes from the sum of three sine waves from three direct digital synthesizers (DDSs). The DDS can be thought of as a digital VCO whose output is programmed by the DSP. The strength of the kick is determined by the $\mathrm{S} / \mathrm{N}$ ratio measured by the Schottky detector. To keep the $\mathrm{S} / \mathrm{N}$ constant, the strength of the kick increases as the energy of the beam increases. In order to kick the same betatron sideband that is measured by the Schottky detector, the DDS output is up converted to $21.4 \mathrm{MHz}$ before being amplified and sent to a stripline kicker.

(4) External Control. The tune tracker communicates with the control system via Ethernet. The control system is able to read the locked tunes found by the tune tracker as well as to allow a user to set it up remotely. An important parameter of each PLL is the loop gain. This can be changed by setting the gain multiplier $g$ for each PLL. The type of excitation can also be changed or turned on and off by the user. The original intention was to use all three DDSs with each locking to a different synchrotron sideband so that a weighted average of the locked frequencies is used to calculate the actual tune. For the experiments discussed in this paper, only one of the DDSs is turned on. 


\subsection{Other Parameters}

The closed loop bandwidth of the continuous PLL has been measured and is dependent on the gain multiplier $g$ shown in Figure 4. It ranges from 2 to $7.5 \mathrm{~Hz}$ for $g=1$ to 10 . The closed loop bandwidth gives information on how long it takes the PLL to lock when there is a step in the tune frequency.

The maximum rate at which the tune data can be sent back to the control system is about $10 \mathrm{~Hz}$. This rate is determined by the handshaking overhead between the control system and the PLL. Internally, each PLL updates at a frequency of $100 \mathrm{kHz}$.

\section{Beam Measurements}

There are three measurements that will be discussed in this section. The first measurement compares the continuous and pulsed PLL methods. This measurement will confirm that the pulsed PLL will find the centre of the betatron tune while the continuous PLL will lock to a phase null which is not necessarily at the centre. The second measurement shows tracking when the tune of the Tevatron is changed. And finally, emittance growth which is

important for operating the tune tracker during HEP operations is also looked at. 


\subsection{Comparison between Continuous and Pulsed PLL}

As have been discussed in the Pulsed Excitation section, by pulsing the excitation, the observed phase response is smoother than continuous excitation. What remains is to show that the pulsed PLL will indeed lock close to the middle of the betatron tune, i.e. at one of the crosses shown in Figure 5. In order to demonstrate this, at the start of the experiment both continuous and pulsed PLLs are locked to the centre of the betatron tune at $A$ and at $C$ respectively. The PLL excitation frequency is then yanked by $+350 \mathrm{~Hz}(4$ synchrotron lines) to cause the PLL to lose lock. After a while, the continuous PLL eventually locks to phase null $B$ while the pulsed PLL locks to a place that is close to the centre of the betatron tune at $D$. The PLL excitation frequency is then yanked by $-500 \mathrm{~Hz}$ (6 synchrotron lines) and again both PLLs eventually lock. Figure 7 shows the results of this experiment. It is interesting that this effect is not symmetric about the centre. This is easily understood by looking again at the phase response shown in Figure 5. For the continuous PLL when the initial lock is at $A$ and after the excitation is yanked y $+350 \mathrm{~Hz}$, there is only one nice zero crossing to the right at $B$. While yanking to lower frequencies, there are no zero crossings other than the one at $A$. The same argument applies for the pulsed PLL.

\subsection{Tracking}

This section shows the tune tracker in action for three cases. The first example shown in Figure 8 demonstrates how the tune tracker follows the motion of the tunes when the air-core quadrupoles are modulated by a sine 
wave. The tunes measured by the tune tracker are used to calibrate the air-core quadrupoles for beta function measurements in the Tevatron. See Figure 8.

The second and third experiments show the data taken from HEP stores 4490 and 4492. See Figures 9 and 10. Data is taken for both continuous and pulsed PLL when the beam in the Tevatron is ramped and and squeezed. One interesting observation is that although these two stores are only two days apart, the tunes are quite different especially at the end of the squeeze at $\mathrm{LBSEQ}=25$. The difference in tunes between the two stores at this point is about $\Delta Q_{x, y}=0.001$. For store 4492 , the tunes landed at a point in the tune plane which caused bad beam lifetime and high losses at the experiments. The cure was to move these tunes higher and closer to the tunes like that in store 4490. The cause of the tune drift is suspected to come from slow rolls of the low beta quadrupoles at the experiments.

\subsubsection{Accuracy}

At flattop, the measured accuracy of both the pulsed PLL and continuous PLL can be compared for different gain multipliers. This is shown in Table 1. In practice, the gain multiplier has been set to values so that the PLL can

respond quickly to changes in tune and with rms error better than $1 \times 10^{-4}$ tune units.

\subsection{Emittance Growth}

One possible weakness of this method is emittance growth because the beam must be coherently excited. Measurements of emittance have been taken for 
10 minutes at injection energy and when the beam is taken up the ramp and through the squeeze for HEP. There is no measurable emittance growth measured by the Tevatron flying wires when the maximum power of the kickers are capped at $180 \mathrm{~mW}$ each.

\section{Conclusion}

The tune tracker system at Fermilab has been successfully shown to work up the ramp and through the squeeze for both continuous and pulsed PLLs. The system has been turned on for routine HEP operations and the tracking data has been used to tune the Tevatron and to diagnose problems which arise when the tunes end up in the wrong place. The tune tracker has also provided a historical record of the HEP tunes in the Tevatron which has highlighted the importance of preventing the tunes from drifting. In the future, the PLL system will form the basis for doing both chromaticity and coupling tracking as well as the possibility of doing tune feedback.

\section{Acknowledgements}

The author wishes to thank V. Shiltsev, P. Cameron, K. Koch, J. Steimel and J. Marriner for useful discussions and the Tevatron group for their support.

\section{References}

[1] P. Cameron et al, RHIC Third Generation PLL Tune System, Proceedings of the Particle Accelerator Conference 2003, p. 524-526. 
[2] C.Y. Tan, The Tevatron Tune Tracker PLL - Theory, Implementation and Measurements, Fermilab Technical Memo TM-2275, 2004.

[3] D. Martin et al, A Resonant Beam Detector for Tevatron Tune Monitoring, Accelerator Science and Technology Proceedings, v. 3, p. 1486-1488, 1989. 
TRACE A: Freq Response

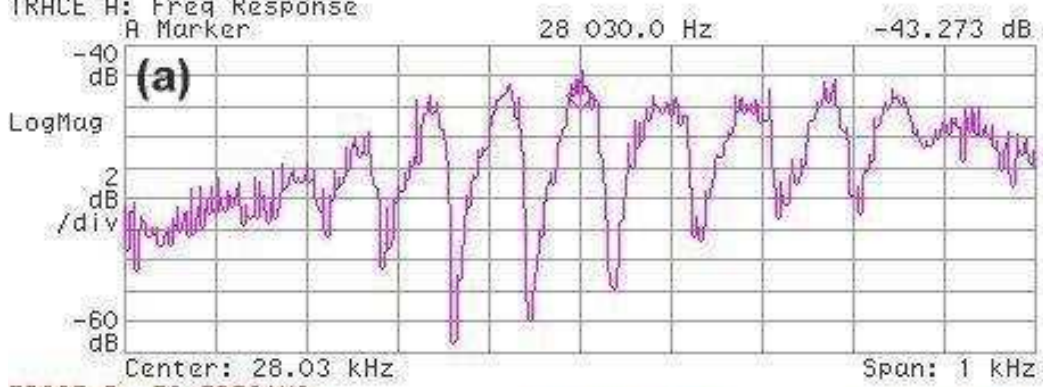

TRACE B: F2 FRES*K2

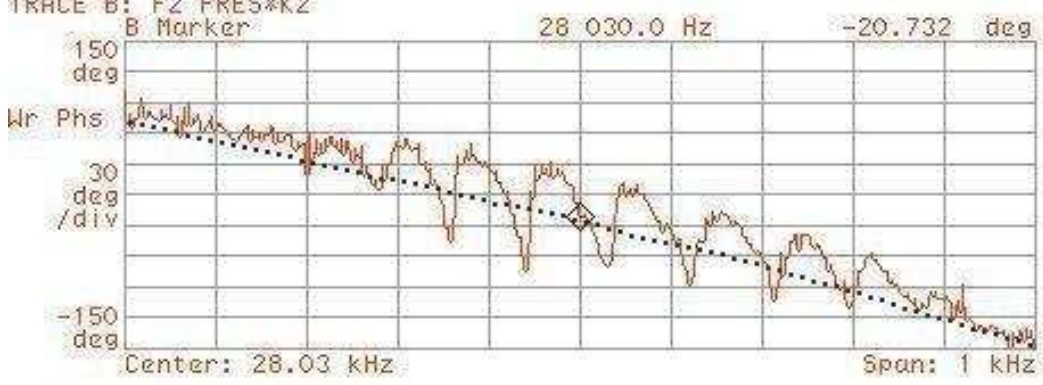

TRACE A: Freq Response

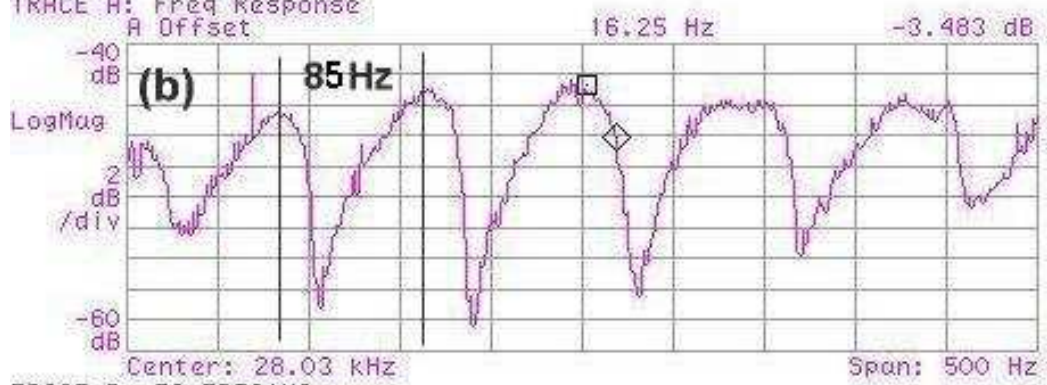

TRACE B: F2 FRES*K2

TRACE B: F2 FRES*

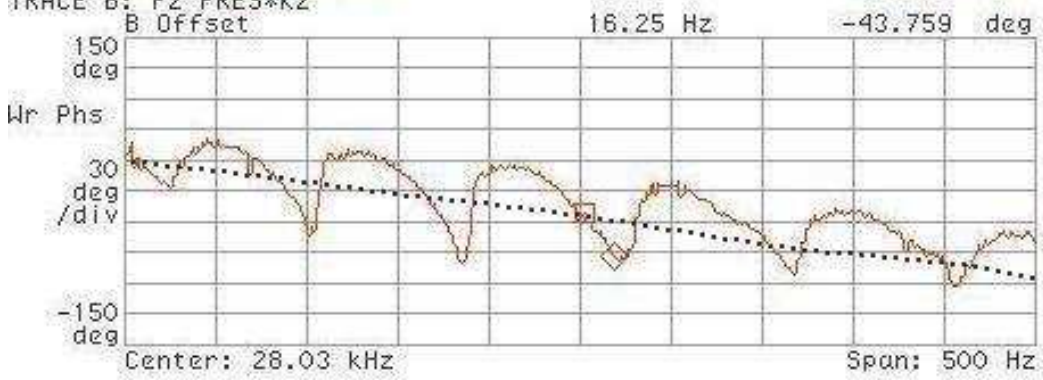

Fig. 1. The horizontal frequency response in (a) and (b) of the beam clearly shows the synchrotron phase nulls superimposed on the betatron phase response (dotted lines). (b) is the zoomed in view which shows that the synchrotron lines are separated by $85 \mathrm{~Hz}$. 

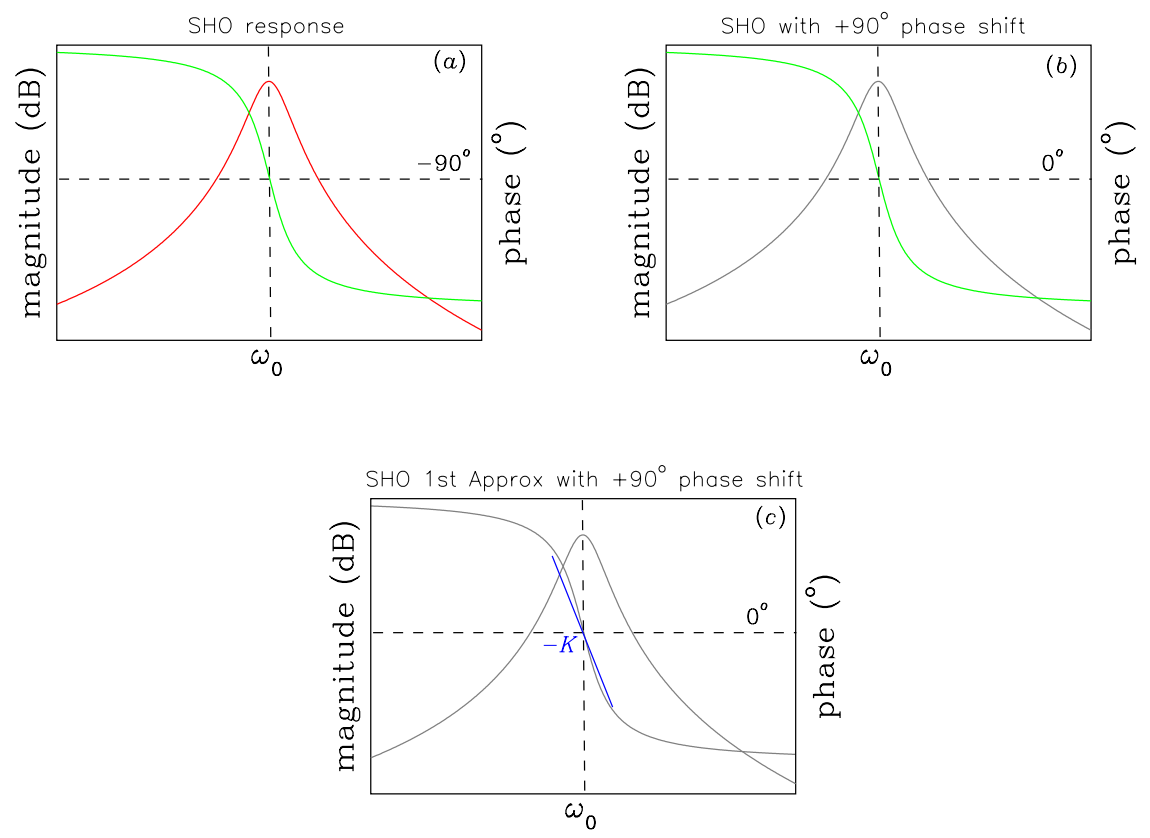

Fig. 2. The stages of how the beam phase response approximation is obtained.

Block Diagram of PLL

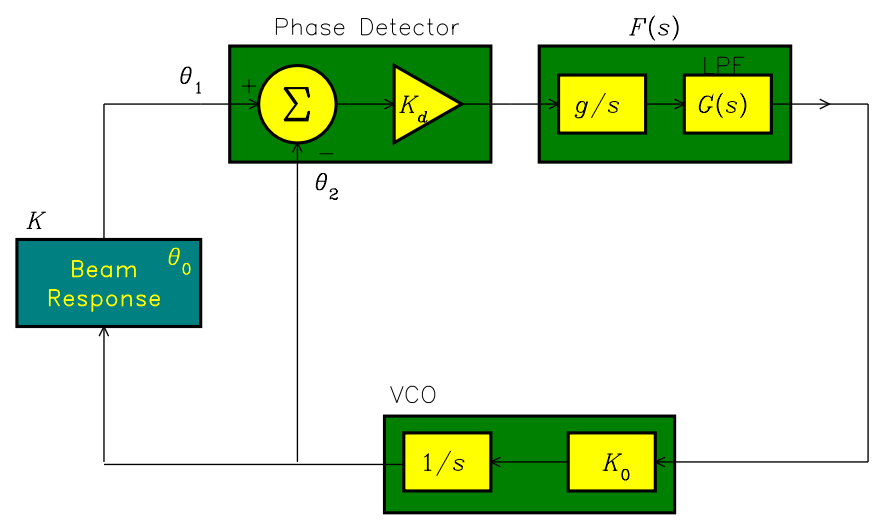

$\mathscr{H}=\frac{\theta_{2}}{\theta_{0}}=\frac{g K_{0} K_{d} K G(s)}{s+g K_{0} K_{d} K G(s)}$

Fig. 3. This is the block diagram of the PLL. 


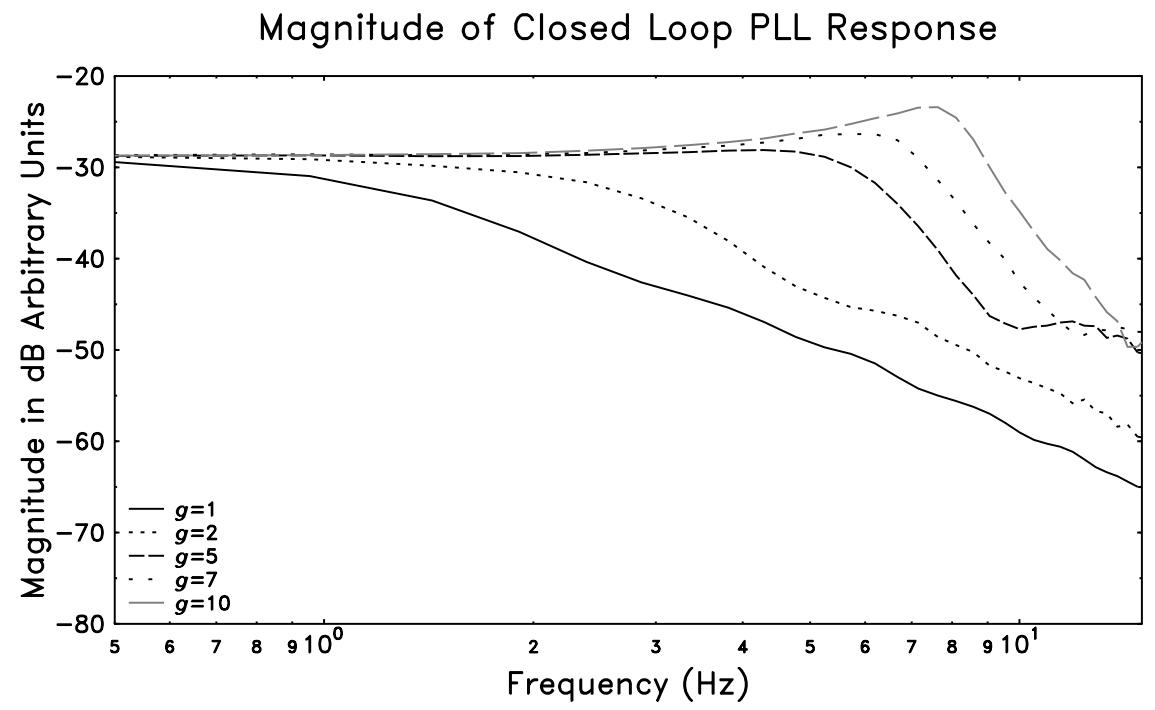

Fig. 4. The magnitude of the closed loop frequency response of the PLL.

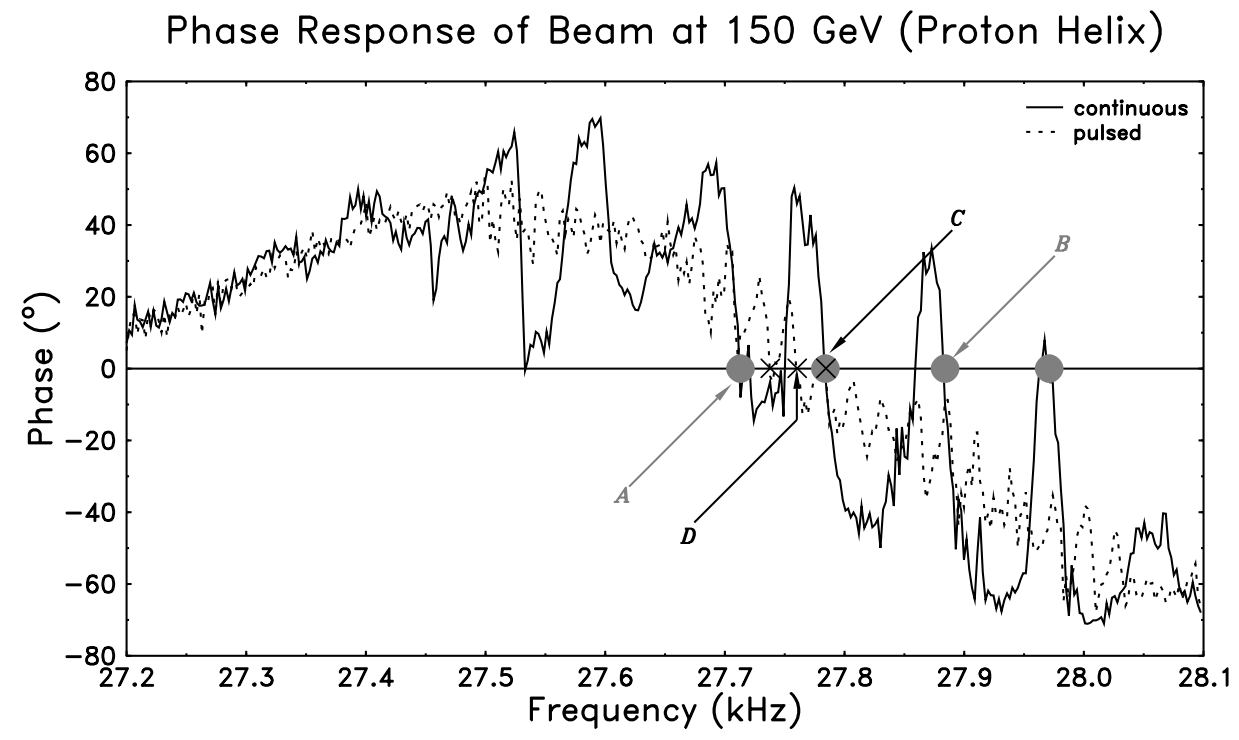

Fig. 5. The horizontal phase response for a bunch of protons on the helix at $150 \mathrm{GeV}$ with continuous and pulsed excitation. The $\bullet$ and $\times$ show the possible places where the PLL can lock to. 


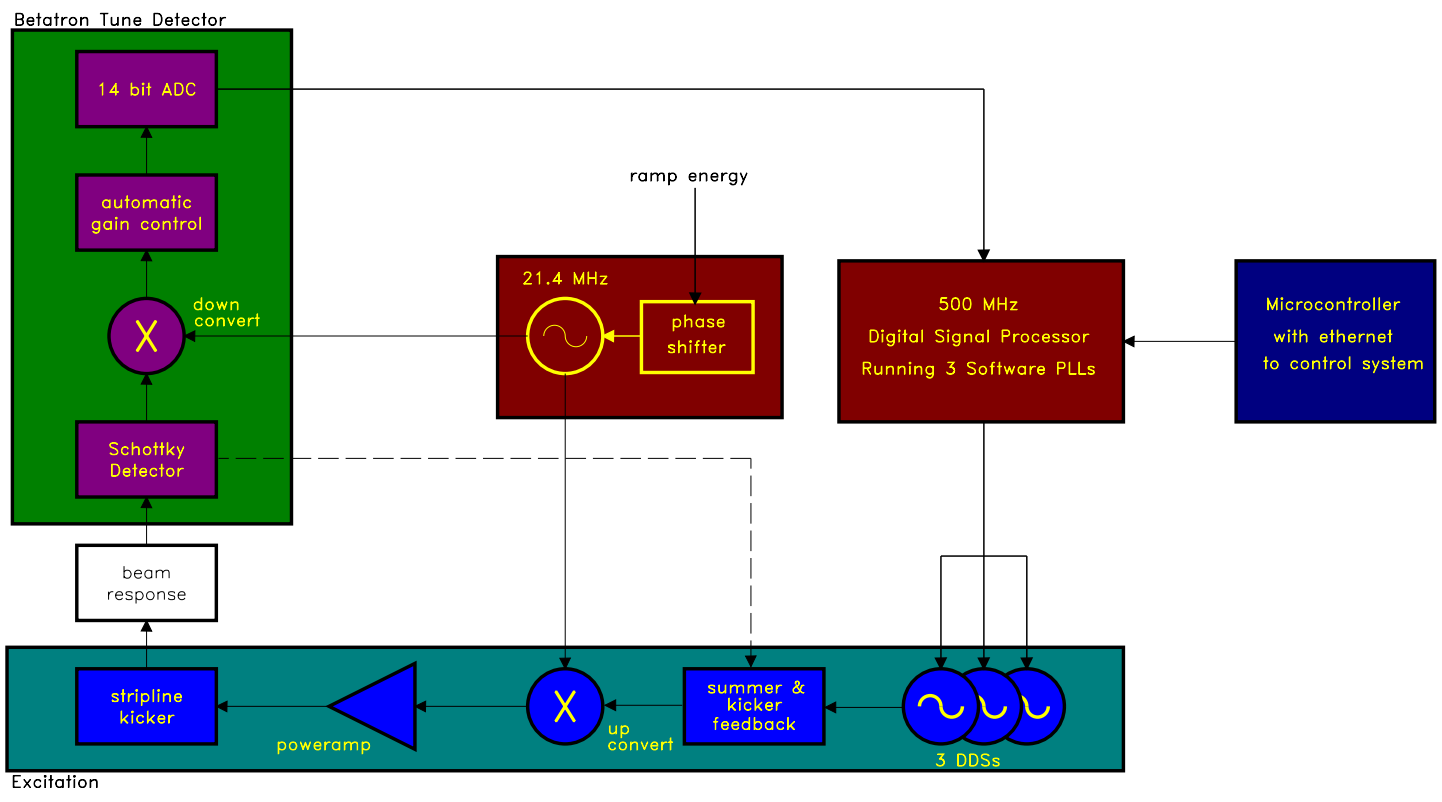

Fig. 6. The block diagram of the implementation.

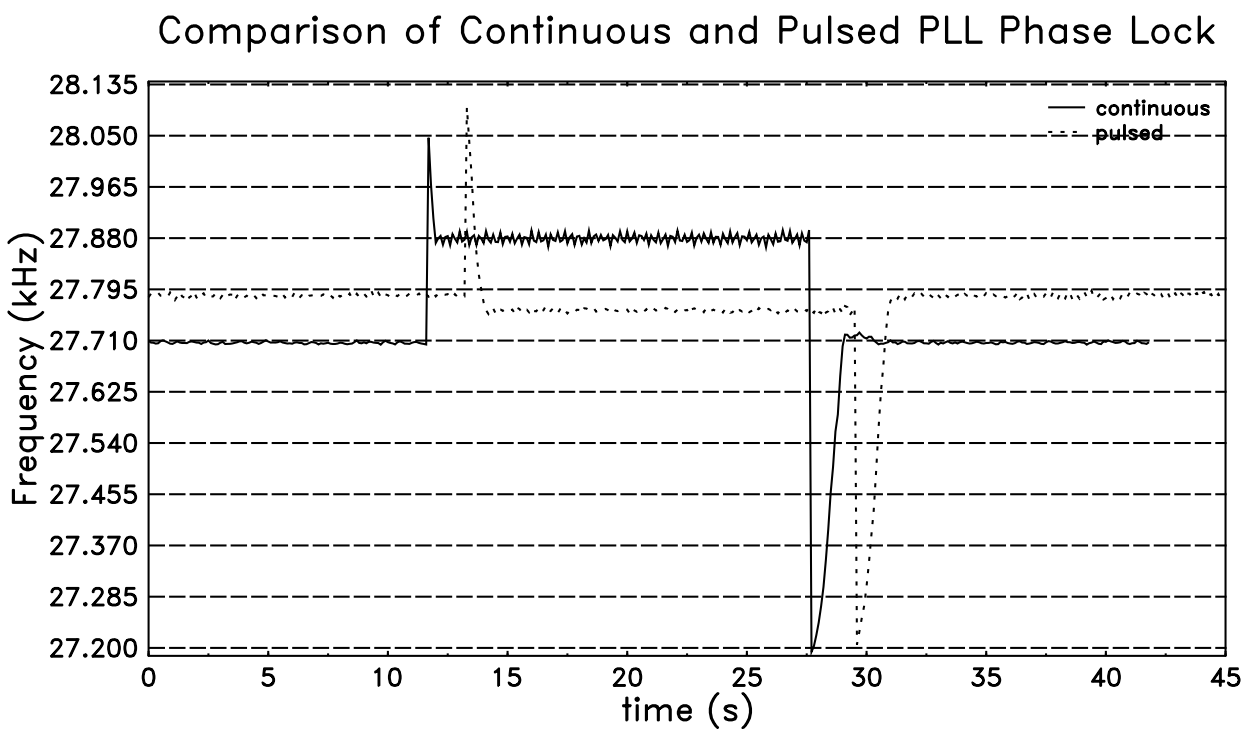

Fig. 7. This compares the continuous PLL and pulsed PLL when the lock frequency is yanked by $+350 \mathrm{~Hz}$ and $-500 \mathrm{~Hz}$ from $27.71 \mathrm{kHz}$. The distance between the horizontal dotted lines is $85 \mathrm{~Hz}$ which is the synchrotron frequency at $150 \mathrm{GeV}$. 


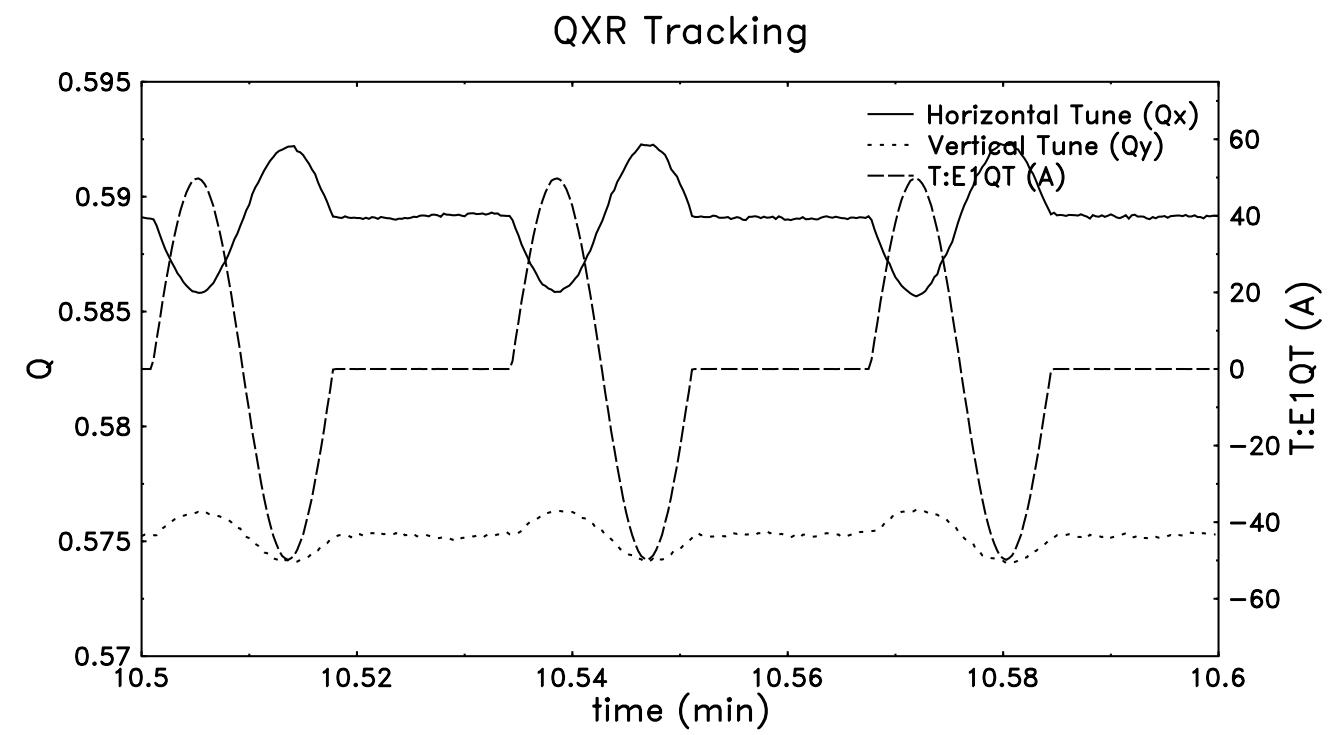

Fig. 8. The tune tracker tracks both the horizontal and vertical tunes as the current in the air core QXR quadrupole T:E1QT is changed. 
Tracking Up the Ramp

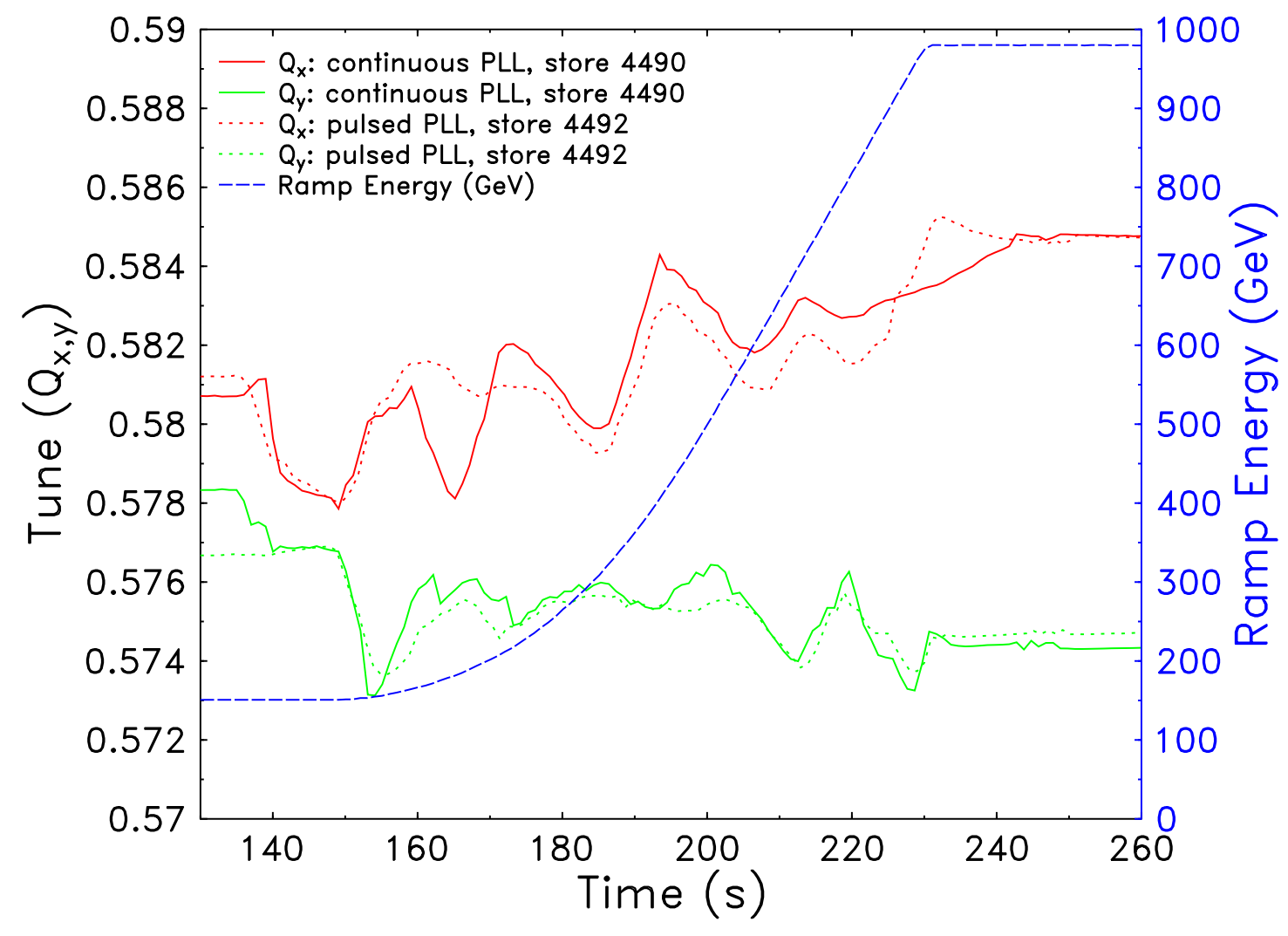

Fig. 9. Tracking with continuous PLL up the ramp for HEP store 4490 and pulsed PLL for HEP store 4492. $Q_{x}$ is the horizontal tune and $Q_{y}$ is the vertical tune. 


\section{Tracking through the Squeeze}

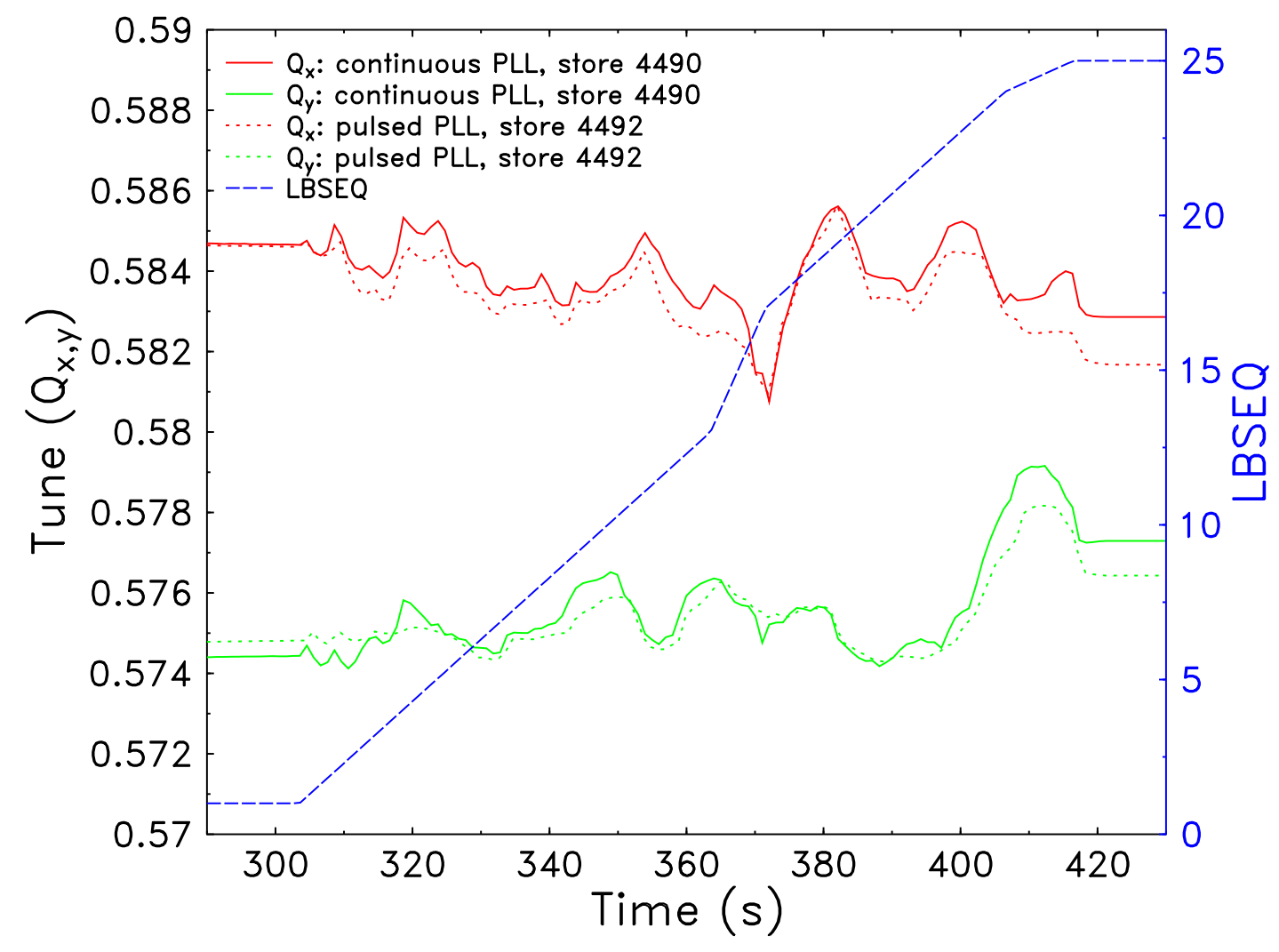

Fig. 10. Tracking using continuous PLL going through the squeeze for HEP store 4490. LBSEQ (Low Beta Sequence) are the steps of the squeeze. 
Table 1

Tracking Error at Flattop

\begin{tabular}{c|c|c}
\hline PLL type & Gain Multiplier & RMS error (tune units) \\
\hline Continuous & 4 & 0.0003 \\
& 2 & 0.0001 \\
\hline \hline Pulsed & 16 & 0.0006 \\
& 1 & 0.00008 \\
\hline \hline
\end{tabular}

